\title{
Altered Expression of Autophagy-related Genes in Human Colon Cancer
}

\author{
Shawn Gurwara ${ }^{1}$, Themistoklis Kourkoumpetis ${ }^{2,3}$, Liang Chen ${ }^{2,3}$, Fengju Chen ${ }^{1,4}$, David Y. Graham ${ }^{2}$, \\ N. Tony Eissa ${ }^{5}$, Michael Ittmann 6 , Hashem B. El-Serag ${ }^{2,3,4}$ and Li Jiao $2,3,4,7^{*}$ \\ ${ }^{1}$ Department of Medicine, Baylor College of Medicine, Houston, TX, USA; ${ }^{2}$ Section of Gastroenterology and Hepatology, Baylor College \\ of Medicine, Houston, TX, USA; ${ }^{3}$ Center for Innovations in Quality, Effectiveness and Safety (IQuESt), Michael E. DeBakey VA Medi- \\ cal Center, Houston, TX, USA; ${ }^{4}$ Dan L Duncan Cancer Center, Baylor College of Medicine, Houston, TX, USA; ${ }^{5}$ Section of Pulmonary, \\ Department of Medicine, Baylor College of Medicine, Houston, TX, USA; ${ }^{6}$ Department of Pathology and Immunology, Baylor College of \\ Medicine, Houston, TX, USA; ${ }^{7}$ Center for Translational Research on Inflammatory Diseases (CTRID), Michael E. DeBakey VA Medical \\ Center, Houston, TX, USA
}

\begin{abstract}
Background and objectives: Autophagy is a physiologic mechanism, which utilizes the self-digestion of cell organelles to promote cellular homeostasis, such as in the setting of dysfunctional cellular components, cellular stress or energy-deprived states. In vitro studies have pointed toward the key role of autophagy in colorectal cancer. However, in vivo studies from human colorectal cancer tissues are lacking.

Methods: We collected tissue samples from six patients with colon cancer who received curative surgery at Baylor College of Medicine. We also obtained normal colonic mucosa biopsy from five unrelated polyp-free individuals who were matched to cases individually by age, sex, ethnicity, and colon segment. Total RNA was successfully extracted from fresh frozen tissue biopsies of five tumor tissues and five unrelated normal tissues. We tested the expression levels of 84 genes in a predefined autophagy pathway using the RT ${ }^{2}$ Profiler PCR array. We compared differences using Student's $t$-test. The false-discovery rate was used for multiple testing adjustment. We also used the TCGA dataset to validate our findings.
\end{abstract}

Results: We observed significant differential expression between colon cancer tissue and normal colon mucosa for 29 genes in the autophagy pathway $(p<0.05)$. After multiple testing adjustment, the expression of 17 genes was significantly down-regulated, with fold-change greater than 2 in colon cancer; these included ATG4A, ATG4C, ATG4D, and CTSS $(q<0.10)$. The down-regulation was observed in both early and late stage colon cancer. We observed the same down-regulation of multiple autophagy-related genes using the TCGA data. The ATG9B gene was the only statistically non-significantly up-regulated gene after multiple testing adjustment.

Conclusions: This pilot study showed the down-regulation of multiple autophagy pathway genes in human colon cancer, corroborating the increasing clinical relevance of autophagy in human colorectal carcinogenesis. This preliminary finding should be validated in larger studies.

Keywords: Autophagy; Gene expression; Colorectal cancer; ATG4; Cathepsin Abbreviations: ATG, autophagy-related genes; CD, Crohn's disease; CRC, colorectal cancer; FC, fold change; FR, fold regulation; FDR, false discovery rate; IHC, immunohistochemical; SNP, single nucleotide polymorphism.

Received: May 18, 2018; Revised: June 25, 2018; Accepted: July 06, 2018

"Correspondence to: Li Jiao, Center for Innovations in Quality, Effectiveness and Safety (IQuESt), Michael E DeBakey VA Medical Center, 2002 Holcombe Blvd, MS152, Houston 77030, TX, USA. Tel: 713-440-4456; Fax: 713-748-7359; E-mail: jiao@bcm.edu

How to cite this article: Gurwara S, Kourkoumpetis T, Chen L, Chen F, Graham DY, Eissa NT, Ittmann M, El-Serag HB, Jiao L. Altered Expression of Autophagy-related Genes in Human Colon Cancer. Exploratory Research and Hypothesis in Medicine 2018;3(3):47-53. doi: 10.14218/ERHM.2018.00007.
Introduction

Colorectal cancer (CRC) is one of the major causes of death worldwide. The carcinogenic processes of CRC involve complex mechanisms. Autophagy is a physiologic mechanism that utilizes self-digestion of cell organelles to promote cellular homeostasis in the setting of dysfunctional cellular components, cellular stress or energy-deprived states. An extensive network of proteins coordinates the process of autophagy. ${ }^{1}$

Autophagy can play an instrumental role in the pathogenesis 
of CRC. ${ }^{1}$ Autophagy reduces carcinogenesis and acts as a tumor suppressor by removing damaged organelles and reducing reactive oxygen species and DNA damage, and by promoting autophagic cell death. However, in established tumors, autophagy promotes cancer growth by providing tumor cells access to nutrients for growth and metabolism as well as increasing drug resistance and ensuring the maintenance of the cancer stem cell. ${ }^{2}$ Autophagyrelated genes (ATGs) play an integral role in the complex cellular pathway of autophagy. Thirty-six such genes have been identified to date. ${ }^{3}$ Early clinical trials have shown the potential benefit of inhibiting parts of the autophagy pathway in many other cancers, including pancreatic cancer, multiple myeloma, and melanoma. ${ }^{3}$ Novel clinical biomarkers are being discovered via ongoing clinical studies to monitor autophagy in patients. ${ }^{4}$

However, little is known about the expression of autophagy genes in human colon cancer. ${ }^{5,6}$ In this study, we compared the expression levels of $A T G$ between normal and colon cancer tissues using a predefined autophagy pathway array. We hypothesized that the genes involved in autophagy were differentially expressed in colon tumor tissue versus normal control tissues.

\section{Methods}

\section{Study participants}

The study population and research approaches were described previously. ${ }^{7}$ Our study was conducted at the affiliated hospitals of Baylor College of Medicine (BCM) and involved six patients who were diagnosed with colon cancer and subsequently underwent total curative surgical excision during the years of 2007-2013. None received pre-operative neoadjuvant chemotherapy. The resected tumor tissues and normal adjacent mucosa samples were snap-frozen in liquid nitrogen and stored in $-80^{\circ} \mathrm{C}$. Additionally, we acquired colon mucosa samples from five unrelated patients who underwent a colonoscopy and were shown to have normal colons endoscopically at the Michael E. DeBakey Veterans Affairs Medical Center (MEDVAMC). These samples served as the control samples when the RNA quality of normal adjacent samples was not adequate for gene expression analysis. The tumor and its unrelated control tissue were matched in relation to colonic segment, race/ethnicity, age ( \pm 5 years), and sex. All patients provided informed consent to participate in a research and use their samples. The Institutional Review Boards of MEDVAMC and BCM approved the study protocol.

\section{$R N A$ extraction and gene expression analysis}

In the genomic core lab at the Texas Medical Center Digestive Disease Center, using the NucleoSpin RNA isolation kit (MACHEREY-NAGEL Inc, Bethlehem, PA, USA), we obtained total RNA from 17 fresh frozen tissue biopsy samples (six biopsies from excised cancerous tissues, six biopsies from adjacent normal colon mucosa, and five from unrelated normal control mucosa). The RNA purity of samples was confirmed with a nucleic acid 260:280 ratio, which was above 2.0 for all. Nonetheless, for one tumor sample and five normal adjacent mucosa samples, the RNA integrity number (RIN) was $<6.0$. These samples were ultimately excluded from the experiment as they were deemed not appropriate for gene expression analysis. Thus, our analysis was restricted to the samples from five mucosal specimens with colon cancer and five biopsies from matched unrelated healthy controls. ${ }^{7}$
We utilized the Qiagen $\mathrm{RT}^{2}$ Profiler PCR Array (Valencia, CA, USA) to determine the expression of 84 key genes in the autophagy pathway (primer sequence is available upon request from Qiagen). The array includes genes encoding proteins for autophagic vacuole formation (AMBRA1 (NYW1), ATG12, ATG16L1, ATG4A, ATG4B, ATG4C, ATG4D, ATG5, ATG9A, ATG9B, BECN1, GABARAP, GABARAPL1, GABARAPL2, IRGM, MAP1LC3A, MAP1LC3B, RGS19, ULK1, WIPI1), vacuole targeting proteins (ATG4A, AT$G 4 B, A T G 4 C, A T G 4 D, G A B A R A P)$, transport proteins (ATG10, ATG16L1, ATG16L2, ATG3, ATG4A, ATG4B, ATG4C, ATG4D, ATG7, ATG9A, GABARAP, GABARAPL2, RAB24), autophagosome-lysosome linkage proteins (DRAM1, GABARAP, LAMP1, $N P C 1)$, ubiquitination proteins ( $A T G 3, A T G 7, H D A C 6)$, and proteases $(A T G 4 A, A T G 4 B, A T G 4 C, A T G 4 D)$. At the core facility at $\mathrm{BCM}$, the assays were conducted using the Bio-Rad Lightcycler RealTime PCR system (Bio-Rad, Hercules, CA, USA).

\section{Analysis of mRNA microarray data from TCGA}

We also downloaded mRNA expression data of 244 TCGA (The Cancer Genome Atlas) colon adenocarcinoma and rectum adenocarcinoma (COAD/READ) patients on June 14, 2018 (www. firebrowse.org). This dataset included 22 normal samples and 222 $\mathrm{CRC}$ tissues. Using these data, we examined mRNA expression levels of $A T G 4 A, A T G 4 B, A T G 4 C, A T G 4 D, G A A, G A B A R A P$, $C T S D$, and CTSS genes in the CRC tissues versus the control tissues. The sequencing platform of the mRNA expression data was UNC_IlluminaHiseq RNASeqV2.

\section{Statistical analysis}

The RT ${ }^{2}$ Profiler PCR Array data analysis version 3.5 was utilized for data analysis. The gene expression levels were normalized to $A C T B, G A P D H$, and RPLPO. The Student's $t$-test was used to compare the expression levels between diverse groups (overall cancer versus control; early- or late-stage cancer versus control) utilizing the normalized gene expression data. Fold-change (FC)/fold-regulation (FR) was calculated using the $\Delta \Delta \mathrm{Ct}$ method (wherein $\Delta \mathrm{Ct}$ was calculated between a gene of interest and an average of reference housekeeping genes, after that the $\Delta \Delta \mathrm{Ct}$ calculation was performed: $\Delta \mathrm{Ct}$ (Test Group) $-\Delta \mathrm{Ct}$ (Control Group)). Subsequently, we calculated FC using the $2^{\wedge} \Delta \Delta \mathrm{Ct}$ formula.

To visualize gene expression changes, we used a scatter plot to compare the normalized expression of every gene on the array between the tumor and normal tissues. On the plot, we used the middle line to denote unchanged gene expression. The selected FR threshold was denoted by the dotted lines above or below the middle line. For multiple testing for 84 genes, we adjusted it using the false discovery rate (FDR). An FDR q value $<0.10$ denoted statistical significance. ${ }^{7}$ For the downloaded TCGA data, we performed the Student's $t$-test comparing CRC tissue with normal tissue.

\section{Results}

The five cancer patients with high-quality RNA included in this analysis were all non-Hispanic Caucasian males between 55 and 75 years old, with a mean age of 62 years. Three patients had earlystage tumors, and three patients had late-stage tumors. The characteristics of these study participants have been described. ${ }^{7}$

We observed significant down-regulation of 29 genes in the autophagy pathway $(p<0.05)$ in colon cancer tissues compared to 


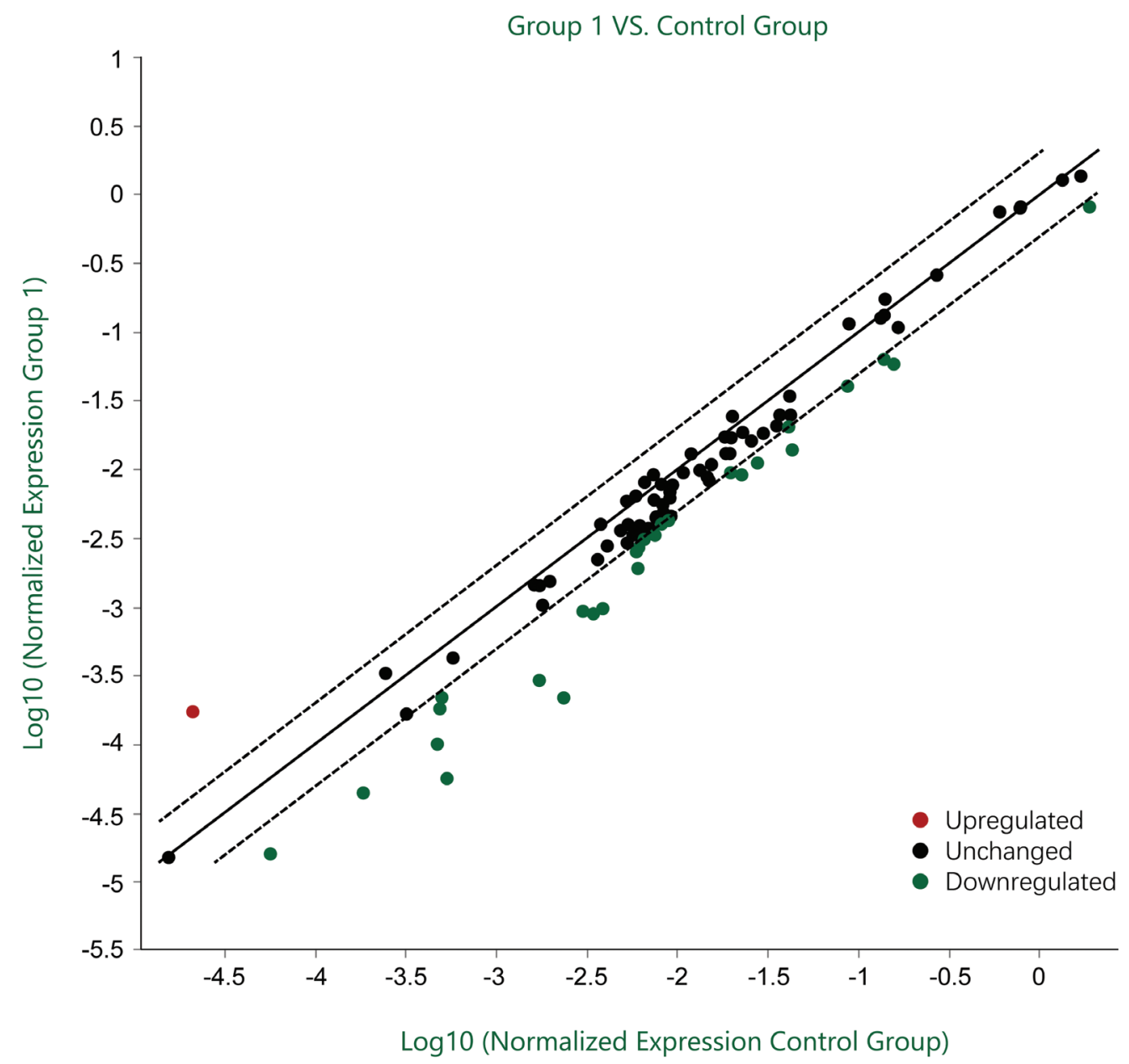

Fig. 1. Scatterplot of autophagy gene expression among colon cancer patients and controls. The dotted lines indicate the selected fold-regulation threshold. Data points beyond the dotted lines in the upper left and lower right sections meet the selected fold-regulation threshold. The figure was generated from the RT² Profiler PCR Array Data Analysis Software, version 3.5.

the normal colonic mucosa (Fig. 1, Supplemental Table S1). The down-regulations were observed for both early- and late-stage tumors. Furthermore, after adjusting for multiple testing, the difference of 17 genes remained statistically significant. The FR was greater than 2 for all 17 genes, including $A T G 4 A, A T G 4 C, A T G 4 D$, CTSD, CTSS, ESR1, GAA, and GABARAP (FDR $q<0.10$; Table 1). $A T G 9 B$ was the only ATG gene that was up-regulated in cancer tissue compared with control tissue $(\mathrm{FC}=8.39, p=0.06)$. However, the up-regulation was statistically nonsignificant after multiple testing adjustment. The differential expression was consistently observed in early- or late-stage tumors (Table 1).

By comparing CRC tissue with normal tissue using the TCGA data, we also found significant down-regulation of $A T G 4 A, A T$ G4C, ATG4D, GAA, GABARAP, CTSD, and CTSS (Fig. 2).

\section{Discussion}

This pilot study suggested differential expression of a number of genes in the autophagy pathway in both early-and late-stage colon cancer compared with normal colonic tissues. Down-regulation of $A T G$ in colon cancer may comprise the host's response in prevent- ing the accumulation of genetic defects that accompany malignant transformations and therefore promote tumor progression. The analysis based on the TCGA data validated our major findings.

We found that multiple ATGs were down-regulated in colon cancer compared to normal mucosa after accounting for multiple testing, including $A T G 4 A, A T G 4 C$, and $A T G 4 D$. ATG4 genes play multiple roles in autophagy, including autophagic vacuole formation and targeting, and proteases. In yeast, the Atg4-family cysteine proteases play a crucial role in preparing Atg8 for conjugation to lipid membranes and for the deconjugation of Atg8 from the autophagosomes. ${ }^{8}$ There are eight human Atg8 homologues that belong to two subfamilies: LC 3 and GABARAP. Atg4a is known to be a potent protease for the GABARAP family but not the LC3 family. ${ }^{9}$ In our study, the GABARAP gene was also significantly down-regulated. Contrary to our results, a recent study on gastric cancer reported the higher expression of $A T G 4 A$ in cancer tissues and metastatic lymph nodes than normal tissues. That study also showed that ATG4A up-regulated the Notch signaling pathwayinduced metastasis in gastric cancer cells. ${ }^{10}$

Of all the ATG4 proteases, ATG4B has been shown to be the most functionally dominant in autophagy regulation in mammals. ${ }^{11}$ Liu et al. ${ }^{12}$ showed that knock-down of the $A T G 4 B$ in CRC 
Table 1. Differential expression of selected autophagy genes in human colon cancer tissues and control tissues

\begin{tabular}{lllllllll}
\hline & Fold-regulation, All tumor & $\boldsymbol{p}$ & $\boldsymbol{q}$ & Fold-regulation, Early tumor & $\boldsymbol{p}$ & Fold-regulation, Late tumor & $\boldsymbol{p}$ \\
\hline PIK3CG & -10.58 & 0.01 & 0.08 & -7.97 & 0.09 & -14.06 & 0.07 \\
IGF1 & -5.84 & 0.0009 & 0.08 & -2.39 & 0.09 & -14.27 & 0.04 \\
TNF & -4.59 & 0.01 & 0.08 & -4.1 & 0.07 & -5.14 & 0.05 \\
ESR1 & -4.05 & 0.01 & 0.08 & -3.18 & 0.08 & -5.15 & 0.05 \\
BCL2 & -3.89 & 0.01 & 0.08 & -3.21 & 0.12 & -4.7 & 0.03 \\
RGS19 & -3.19 & 0.007 & 0.08 & -2.65 & 0.07 & -3.83 & 0.04 \\
GAA & -3.11 & 0.01 & 0.08 & -4.39 & 0.02 & -2.21 & 0.11 \\
TNFSF10 & -3.05 & 0.01 & 0.08 & -2.12 & 0.12 & -4.39 & 0.04 \\
CTSD & -2.63 & 0.00085 & 0.07 & -2.08 & 0.02 & -3.31 & 0.01 \\
PTEN & -2.43 & 0.01 & 0.08 & -2.67 & 0.06 & -2.2 & 0.07 \\
ATG4A & -2.25 & 0.02 & 0.09 & -2.31 & 0.09 & -2.2 & 0.08 \\
ATG4C & -2.31 & 0.01 & 0.10 & -2.40 & 0.07 & -2.22 & 0.06 \\
ATG4D & -2.22 & 0.01 & 0.08 & -2.14 & 0.1 & -2.3 & 0.05 \\
CTSS & -2.16 & 0.01 & 0.08 & -1.78 & 0.11 & -2.64 & 0.05 \\
GABARAP & -2.12 & 0.0003 & 0.07 & -2.09 & 0.03 & -2.15 & 0.17 & -2.65 \\
CASP3 & -2.05 & 0.02 & 0.08 & -1.58 & 0.08 & -2.1 & 3.91 & 0.01 \\
TGFB1 & -2.01 & 0.01 & 0.08 & -1.92 & 0.16 & & 0.03 \\
ATG9B & 8.39 & 0.06 & 0.16 & 18 & 0.22 & \\
\hline
\end{tabular}

cell lines induced autophagic flux and reduced cyclin D1 expression to inhibit G1/S phase transition of the cell cycle. However, in our research, the expression of $A T G 4 B$ was statistically non- significantly down-regulated in tumors, with only a small FC $A T G 4 C$ gene knock-out in mice has been shown to aid in forming methylcholanthrene-induced fibrosarcomas, suggesting a regu-
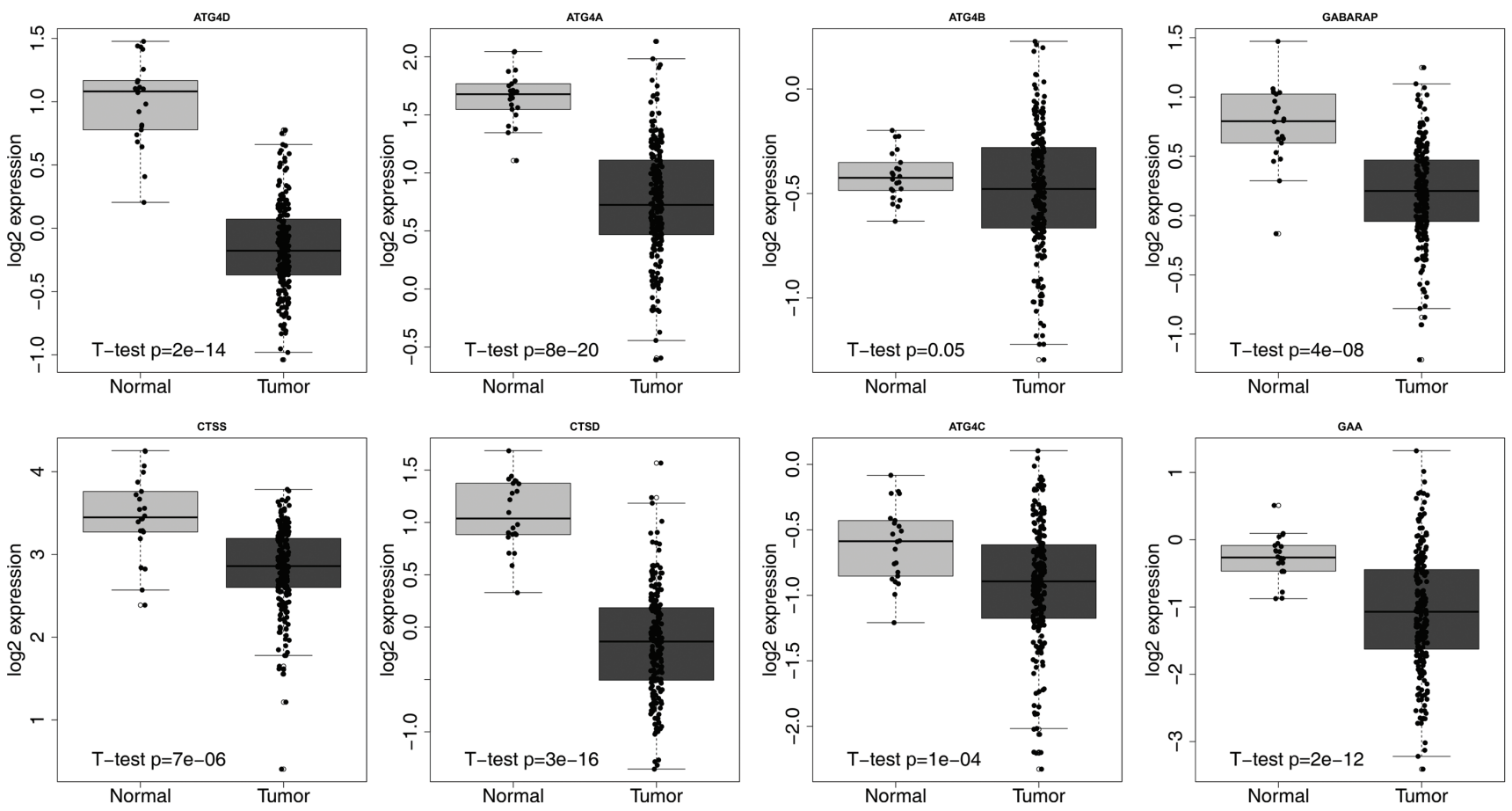

Fig. 2. Box-plot of log2 expression levels of ATG4 genes, GABARAP, CTSS, CTSD and GAA between normal tissue and colorectal cancer tissue, according to the TCGA (The Cancer Genome Atlas) data. 
latory impact on tumor cells. ${ }^{13}$ We found the $A T G 4 C$ gene was down-regulated significantly, as was the $A T G 4 D$ gene. In line with our finding, it has been shown that the expression of the $A T G 4 D$ gene was significantly lower in breast cancer tissues than that in the adjacent normal tissues. ${ }^{14}$ The identification of diminished ATG4 gene expression in colon cancer suggested that the formation of autophagosomes may be compromised in colon cancer. In our study, $A T G 9 B$ was the only gene to be highly up-regulated with an FC of 16. However, the difference between colon cancer and normal mucosa was not statistically significant.

Cathepsin is an aspartic endoprotease that is distributed in all lysosomes in the human body. ${ }^{15}$ Its primary function is to disassemble proteins and activate cascades in prelysosomal compartments. ${ }^{16}$ Cathepsin D (CTSD) and cathepsin S (CTSS) genes were significantly down-regulated in colon cancer compared to normal mucosa after accounting for multiple testing. Contrary results were reported by Sebzda et al. ${ }^{17}$ which showed increased expression of CTSD in colon cancer compared to adjacent normal colon mucosa utilizing immunohistochemical (IHC) staining in 68 patients from Poland. Another study also utilizing IHC testing showed that cathepsins B, L, and D activities were higher in a series of malignant tissues compared with adjacent normal colorectal tissues. ${ }^{18}$ In our study, CTSS was also significantly down-regulated, even after accounting for multiple testing. Our data were incongruous to the prior studies possibly in part because we used mRNA levels to measure gene expression, whereas the prior studies used IHC or enzyme-linked immunosorbent assay. In addition, the function of autophagy genes in cancer development may be tissue-specific.

The research on autophagy and CRC was triggered by a genomewide association study that identified an association between the single nucleotide polymorphism (SNP) rs2241880 of ATG16L1 and risk of inflammatory bowel disease. ${ }^{19}$ Hampe et al. ${ }^{19}$ showed a statistically significantly positive association between this SNP and Crohn's disease in a German population. This SNP was also associated with an improved overall survival in CRC. ${ }^{20}$ However, we did not find the expression of the ATG16L1 to be associated with colon cancer in our sample.

BECN1 (Bectin 1), LC3B (MAP1LC3B), and p62 (SQSTM1) are three genes that have been widely reported in CRC research. They are expressed in normal colon mucosa as well. ${ }^{21} \mathrm{We}$ found $L C 3 B$ and $B E C N 1$ nonsignificantly down-regulated and $p 62$ upregulated in cancer tissues. The loss of expression of BECN1 has been reported for ovarian and esophageal cancers. ${ }^{22,23}$ Contrarily, prior studies showed mRNA and protein expression of BECN1 or LC3 were higher in CRC tissue compared with normal adjacent tissue. ${ }^{24-26}$ p62 is one of five autophagy cargo adaptor molecules that can interact with processed LC3 at the autophagosome. p62 accumulation has been observed in liver cancer progression. ${ }^{27} \mathrm{An}$ IHC study also showed that higher p62 expression was an independent adverse prognostic marker in patients with CRC. ${ }^{28}$ p62 and LC3 also contribute to selective autophagy. ${ }^{29,30}$ For example, mitophagy is a selective mode of autophagy in which mitochondria are targeted for degradation. ${ }^{31}$ Mitophagy affects cell stemness, cell fate determination, inflammation, and DNA damage, and has also been implicated in cancer. ${ }^{32}$ It is likely that these genes can influence colon tumorigenesis through regulating mitophagy. ${ }^{33}$

BECN1 and PTEN are important in inducing autophagy in cancer. PTEN is a tumor suppressor gene in colon cancer and was down-regulated in our research. ${ }^{34}$ The down-regulation of autophagy inducers was in line with the overall down-regulation of autophagy pathway genes in colon cancer. A cross-talk exists between apoptosis and autophagy. BECN1 is also a direct substrate of caspase 3. Several genes related to apoptosis, including CAPS3 and $B C L 2$, were also down-regulated in colon cancer in our study.

This was a preliminary study with multiple limitations. First, it was based on a small sample size and, thus, false negative and false positive findings could have occurred. The stratified analysis by tumor stage was exploratory given a limited sample size. Furthermore, transcription factor EB (TFEB) is probably one of the most important transcription regulators for autophagy and lysosome-related genes. TFEB could modulate the expression of a broad range of lysosomal genes. including CTSD and CSTS ${ }^{35}$ TFEB over-expression has been seen in a variety of cancers. ${ }^{36-38}$ However, this gene was not included in the predefined panel in the present study. Lastly, one study shows that clinical implications of autophagy biomarkers, such as LC3 and p62, in CRC prognosis may depend on K-ras mutation status. ${ }^{39}$ As this was a preliminary study, we did not ascertain K-ras mutation status or evaluate protein expression.

In summary, the findings from our study support the tumor suppressor role of autophagy in colon cancer. In particular, the ATG4 proteases may play an important role in colon cancer development. The precise mechanisms inhibiting or leading to autophagy, and the downstream effectors of autophagy in carcinogenesis need to be fully elucidated in order to identify novel biomarkers for prognosis and target for cancer treatment.

\section{Future research directions}

We found that multiple autophagy-related genes were down-regulated in colon cancer tissues. We will validate our findings using larger clinical sample series and by including IHC staining. We will also identify modifiable factors and metabolic stress factors that can contribute to macroautophagy and mitochondria autophagy dysregulation in colon cancer.

\section{Acknowledgments}

This research was supported by Gillson Longenbaugh Foundation and Golfers Against Cancer Organization (PI: LJ), Cancer Prevention Research Institute of Texas (RP\#140767, PI: LJ), and National Cancer Institute P30 Cancer Center (P30 CA125123) for support of the Human Tissue Acquisition and Pathology Shared Resources. This project was also supported in part by Public Health Service (grant P30DK056338), the expert assistance of Lisa D. White and the Specimen Collection Core E of Texas Medical Center Digestive Diseases Center, and Houston VA HSR\&D Center for Innovations in Quality, Effectiveness and Safety (CIN13-413). Finally, we are thankful for Dr. Lang Rao's comments on this manuscript.

\section{Conflict of interest}

The authors have no conflict of interests related to this publication.

\section{Author contributions}

Study design (LJ), sample acquisition (MI, HBE), data analysis (LC, FC), data interpretation and manuscript writing (SG, TK, DYG, MI, NTE, HBE, LJ), critical intellectual input (SG, TK, LC, MI, NTE, HBE, LJ). 
Supporting information

Supplementary material for this article is available at https://doi. org/10.14218/ERHM.2018.00007.

Supplemental Table S1. Differential expression of all autophagy genes in human colon cancer tissue and normal control tissues.

\section{References}

[1] Kim YS, Shin JH, Bae MK, Lee CY, Kim DJ, Chung KY, et al. Autophagy activity in pulmonary metastatic tumor tissues from colorectal cancer: A pilot study. Yonsei Med J 2014;55:1484-1488. doi:10.3349/ ymj.2014.55.6.1484.

[2] Panda PK, Mukhopadhyay S, Das DN, Sinha N, Naik PP, Bhutia SK. Mechanism of autophagic regulation in carcinogenesis and cancer therapeutics. Semin Cell Dev Biol 2015;39:43-55. doi:10.1016/j.semcdb.2015.02.013.

[3] Levy JMM, Towers CG, Thorburn A. Targeting autophagy in cancer. Nat Rev Cancer 2017;17:528-542. doi:10.1038/nrc.2017.53.

[4] Burada F, Nicoli ER, Ciurea ME, Uscatu DC, loana M, Gheonea DI. Autophagy in colorectal cancer: An important switch from physiology to pathology. World J Gastrointest Oncol 2015;7:271-284. doi:10.4251/ wjgo.v7.i11.271.

[5] Bednarczyk M, Muc-Wierzgon M, Waniczek D, Fatyga E, Klakla K, Mazurek $\mathrm{U}$, el al. Autophagy-related gene expression in colorectal cancer patients. J Biol Regul Homeost Agents 2017;31:923-927.

[6] Gil J PK, Sąsiadek MM. May autophagy be a novel biomarker and antitumor target in colorectal cancer? Biomark Med 2016;10:10811094. doi:10.2217/bmm-2016-0083.

[7] Kourkoumpetis T, Royse KE, Chen L, Ravishankar M, Ittmann M, ElSerag $\mathrm{HB}$, et al. Differential expression of tight junctions and cell polarity genes in huma colon cancer. Explor Res Hypothesis Med 2018;3:14-19. doi:10.14218/ERHM.2017.00036.

[8] Maruyama T, Noda NN. Autophagy-regulating protease Atg4: Structure, function, regulation and inhibition. J Antibiot (Tokyo) 2017:7278. doi:10.1038/ja.2017.104.

[9] Li M, Fu Y, Yang Z, Yin XM. Measurement of the activity of the Atg4 cysteine proteases. Methods Enzymol 2017;587:207-225. doi:10.1016/bs.mie.2016.10.024.

[10] Yang SW, Ping YF, Jiang YX, Luo X, Zhang X, Bian XW, et al. ATG4A promotes tumor metastasis by inducing the epithelial-mesenchymal transition and stem-like properties in gastric cells. Oncotarget 2016;7:39279-39292. doi:10.18632/oncotarget.9827.

[11] Zhang L, Li J, Ouyang L, Liu B, Cheng Y. Unraveling the roles of Atg4 proteases from autophagy modulation to targeted cancer therapy. Cancer Lett 2016;373:19-26. doi:10.1016/j.canlet.2016.01.022.

[12] Liu PF, Leung CM, Chang YH, Cheng JS, Chen JJ, Weng CJ, et al. ATG4B promotes colorectal cancer growth independent of autophagic flux. Autophagy. Autophagy 2014;10:1454-1465. doi:10.4161/auto.29556.

[13] Marino G, Salvador-Montoliu N, Fueyo A, Knecht E, Mizushima N, Lopez-Otin C. Tissue-specific autophagy alterations and increased tumorigenesis in mice deficient in Atg4C/autophagin-3. J Biol Chem 2007;282:18573-18583. doi:10.1074/jbc.M701194200.

[14] Zhang XM, Li HJ, Wang D, Li C, Chen Q, Li CL. [expressions and clinical significance of autophagy-related genes ATG2B, ATG4D, ATG9B in breast carcinoma]. Sichuan Da Xue Xue Bao Yi Xue Ban 2016;47:184188. doi:10.13464/j.scuxbyxb.2016.02.009.

[15] Barrett AJ. Cathepsin D. Purification of isoenzymes from human and chicken liver. Biochem J 1970;117:601-607. doi:10.1042/bj1170601.

[16] Diment S, Martin KJ, Stahl PD. Cleavage of parathyroid hormone in macrophage endosomes illustrates a novel pathway for intracellular processing of proteins. J Biol Chem 1989;264:13403-13406.

[17] Sebzda T, Saleh Y, Gburek J, Andrzejak R, Gnus J, Siewinski M, et al. Cathepsin D expression in human colorectal cancer: Relationship with tumour type and tissue differentiation grade. J Exp Ther Oncol 2005;5:145-150.

[18] Adenis A, Huet G, Zerimech F, Hecquet B, Balduyck M, Peyrat JP. Cath- epsin B, L, and D activities in colorectal carcinomas: Relationship with clinico-pathological parameters. Cancer Lett 1995;96:267-275.

[19] Hampe J, Franke A, Rosenstiel P, Till A, Teuber M, Huse K, et al. A genome-wide association scan of nonsynonymous SNPs identifies a susceptibility variant for Crohn disease in ATG16L1. Nat Genet 2007;39:207-211. doi:10.1038/ng1954.

[20] Grimm WA, Messer JS, Murphy SF, Nero T, Lodolce JP, Weber CR, et $a l$. The Thr300ala variant in Atg16l1 is associated with improved survival in human colorectal cancer and enhanced production of type i interferon. Gut 2016;65:456-464. doi:10.1136/gutjnl-2014-308735.

[21] Groulx JF, Khalfaoui T, Benoit YD, Bernatchez G, Carrier JC, Basora $\mathrm{N}$, et al. Autophagy is active in normal colon mucosa. Autophagy 2012;8:893-902. doi:10.4161/auto.19738.

[22] Minamoto T, Nakayama K, Nakamura K, Katagiri H, Sultana R, Ishibashi T, et al. Loss of beclin 1 expression in ovarian cancer: A potential biomarker for predicting unfavorable outcomes. Oncol Lett 2018;15:1170-1176. doi:10.3892/ol.2017.7379.

[23] Weh KM, Howell AB, Kresty LA. Expression, modulation, and clinical correlates of the autophagy protein Beclin-1 in esophageal adenocarcinoma. Mol Carcinog 2016;55:1876-1885. doi:10.1002/mc.22432.

[24] Li BX, Li CY, Peng RQ, Wu XJ, Wang HY, Wan DS, et al. The expression of beclin 1 is associated with favorable prognosis in stage IIIB colon cancers. Autophagy 2009;5:303-306. doi:10.4161/auto.5.3.7491.

[25] Ahn CH, Jeong EG, Lee JW, Kim MS, Kim SH, Kim SS, et al. Expression of beclin-1, an autophagy-related protein, in gastric and colorectal cancers. APMIS 2007;115:1344-1349. doi:10.1111/j.16000463.2007.00858.x.

[26] Shuhua W, Chenbo S, Yangyang L, Xiangqian G, Shuang H, Tangyue $\mathrm{L}$, et al. Autophagy-related genes Raptor, Rictor, and Beclin1 expression and relationship with multidrug resistance in colorectal carcinoma. Hum Pathol 2015;46:1752-1759. doi:10.1016/j.humpath.2015.07.016.

[27] Takamura A, Komatsu M, Hara T, Sakamoto A, Kishi C, Waguri S, et al. Autophagy-deficient mice develop multiple liver tumors. Genes Dev 2011;25:795-800. doi:10.1101/gad.2016211.

[28] Nakayama S, Karasawa H, Suzuki T, Yabuuchi S, Takagi K, Aizawa T, et al. p62/sequestosome 1 in human colorectal carcinoma as a potent prognostic predictor associated with cell proliferation. Cancer Med 2017;6:1264-1274. doi:10.1002/cam4.1093.

[29] Lamark T, Svenning S, Johansen T. Regulation of selective autophagy: The p62/SQSTM1 paradigm. Essays Biochem 2017;61:609-624. doi:10.1042/EBC20170035.

[30] Strappazzon F, Nazio F, Corrado M, Cianfanelli V, Romagnoli A, Fimia $\mathrm{GM}$, et al. AMBRA1 is able to induce mitophagy via LC3 binding, regardless of PARKIN and p62/SQSTM1. Cell Death Differ 2015;22:419432. doi:10.1038/cdd.2014.190.

[31] Zhang W, Ren H, Xu C, Zhu C, Wu H, Liu D, et al. Hypoxic mitophagy regulates mitochondrial quality and platelet activation and determines severity of I/R heart injury. Elife 2016;5:e21407. doi:10.7554/ elife.21407.

[32] Drake LE, Springer MZ, Poole LP, Kim CJ, Macleod KF. Expanding perspectives on the significance of mitophagy in cancer. Semin Cancer Biol 2017;47:110-124. doi:10.1016/j.semcancer.2017.04.008.

[33] Knoll G, Bittner S, Kurz M, Jantsch J, Ehrenschwender M. Hypoxia regulates trail sensitivity of colorectal cancer cells through mitochondrial autophagy. Oncotarget 2016;7:41488-41504. doi:10.18632/oncotarget.9206.

[34] Chen N, Karantza-Wadsworth V. Role and regulation of autophagy in cancer. Biochim Biophys Acta 2009;1793:1516-1523. doi:10.1016/j. bbamcr.2008.12.013.

[35] Sardiello M, Palmieri M, Ronza Ad, Medina DL, Valenza M, Gennarino $\mathrm{VA}$, et al. A gene network regulating lysosomal biogenesis and function. Science 2009;325:473-477. doi:10.1126/science.1174447.

[36] Fu X, Zhang L, Dan L, Wang K, Xu Y. Expression of TFEB in epithelial ovarian cancer and its role in autophagy. Int J Clin Exp Pathol 2016;9:10914-10928.

[37] Giatromanolaki A, Kalamida D, Sivridis E, Karagounis IV, Gatter KC, Harris AL, et al. Increased expression of transcription factor EB (TFEB) is associated with autophagy, migratory phenotype and poor prognosis in non-small cell lung cancer. Lung cancer 2015;90:98-105. doi:10.1016/j.lungcan.2015.07.008. 
[38] Argani P, Lae M, Hutchinson B, Reuter VE, Collins MH, Perentesis J, et al. Renal carcinomas with the $t(6 ; 11)(p 21 ; q 12)$ : clinicopathologic features and demonstration of the specific alpha-TFEB gene fusion by immunohistochemistry, RT-PCR, and DNA PCR. Am J Surg Pathol 2005;29:230-240. doi:10.1097/01.pas.0000146007.54092.37.
[39] Schmitz KJ, Ademi C, Bertram S, Schmid KW, Baba HA. Prognostic relevance of autophagy-related markers LC3, p62/sequestosome 1 , Beclin-1 and ULK1 in colorectal cancer patients with respect to KRAS mutational status. World J Surg Oncol 2016;14:189. doi:10.1186/ s12957-016-0946-x. 